\title{
Plaque Characteristics of Consecutive 31 Cases Which Cause Acute Myocardial Infarction on Coronary Computed Tomography Angiography
}

\author{
Shinichiro Fujimoto, ${ }^{1,2}$ Takeshi Kondo, ${ }^{1}$ Kazuhisa Takamura, ${ }^{1,2}$ Yuko Kawaguchi, ${ }^{2}$ \\ Rie Matsumori, ${ }^{2}$ Makoto Hiki, ${ }^{2}$ Katsumi Miyauchi, ${ }^{2}$ Hiroyuki Daida ${ }^{2}$
}

\begin{abstract}
Background: It has been reported that various types of vulnerable plaque can cause acute myocardial infarction (AMI). The diagnosis of vulnerable plaques using coronary computed tomography angiography (CCTA) has not been established. We evaluated the plaque characteristics on CCTA in subjects who developed AMI more than 30 days after the CCTA was performed. Methods and Results: The subjects were 31 consecutive patients ( $M / F=29 / 2,67 \pm 10 \mathrm{yrs}$, period to AMl onset, $559 \pm 490$ days, known CAD: 16) who developed AMI more than 30 days after the CCTA examination. Culprit lesions were observed in the LMT in 2 patients, RCA in $11, \operatorname{LAD}$ in 12 and LCX in 6 . No significant stenosis $(\leq 50 \%)$ could be found in $24(77.4 \%)$ patients. Positive remodeling (PR: remodeling index $>1.1)$ was found in $24(77.4 \%)$ patients and low-density plaque (LDP: CT values $\leq 50 \mathrm{HU}$ ) was found in 17 (54.8\%). Calcification was spotty in 9 patients, moderate in 12 , severe in 4 and absent in 6 . The period to AMI onset tended to be shorter in patients with $\geq 75 \%$ stenosis, and the patients in the $\mathrm{PR}(+)$ group were significantly younger. Vulnerable plaques identified by CCTA were classified into 4 types: Type I (PR(+) and LDP $(+))$ in $17(54.8 \%)$ patients, Type II $(\mathrm{PR}(+)$ and $\operatorname{LDP}(-))$ in 7 (22.6\%), Type III (PR(-) and LDP(-)) in $4(12.9 \%)$, and Type IV (PR(-) and severe calcification) in $3(9.7 \%)$. Conclusions: AMI arose from plaques with various manifestations on CCTA, but as many as about half of the AMl events were derived from Type I $(\mathrm{PR}(+)$ and $\operatorname{LDP}(+))$ plaques in younger patients.

KEY WORDS: coronary CT angiography, vulnerable plaque, positive remodeling, low- density plaque, acute myocardial infarction
\end{abstract}

\section{Introduction}

Thin cap fibroatheroma, a rupture-prone plaque established to contribute to the histopathology of about $60 \%$ of cases with acute coronary syndrome (ACS), is now recognized as a vulnerable plaque. Narula et al. reported the following features of rupture-prone vulnerable plaques: positive vascular remodeling; plaque accounting for more than $50 \%$ of the blood vessel cross section; necrotic core accounting for more than $25 \%$ of the plaque; appearance of vasa vasorum within the plaque, macrophage infiltration into the fibrous cap; plaque thickness accounting for less than $65 \mu$ of the fibrous cap; and matrix metalloproteinase expression. ${ }^{1)}$ Coronary computed tomography angiography (CCTA) studies by Motoyama et al. revealed the following: first, that ACS-causing plaques have spotty calcification, positive remodeling, and properties consistent with a lowattenuation plaques; ${ }^{2)}$ second, that patients with two-feature

${ }^{1}$ Department of Cardiology, Takase Clinic, ${ }^{2}$ Department of Cardiology, Juntendo University Graduate School of Medicine, 2-1-1 Hongo, Bunkyo-ku, Tokyo, 113-8421, Japan

Received November 17, 2014; Accepted March 11, 2015

doi: 10.7793 j jcoron.21.14-00043 positive plaques, that is, plaques with both positive remodeling and low-attenuation plaque content, have a $22 \%$ probability of developing ACS within 2 years. ${ }^{3)}$ Another investigator showed that non-calcified plaques were more common in ACS patients than in patients with stable angina pectoris. ${ }^{4)}$ Kashiwagi et al. recently reported that CCTA findings of positive remodeling, low-density plaque, and napkin-ring sign were predictive of the detection of thin-cap fibroatheroma by optical coherence tomography. ${ }^{5)}$ CCTA findings of positive remodeling, low CT values, non-calcified or spotty calcification, and napkin-ring sign are now regarded as the defining features of a ruptureprone vulnerable plaque. ${ }^{2-6)}$

Naghavi et al., however, reported that various types of vulnerable plaque can cause ACS and sudden cardiac death. ${ }^{7)}$ Pathologically, the frequency of coronary lesions with ACScausing thrombi is $55-60 \%$ for ruptures, $30-35 \%$ for erosions, and $3-7 \%$ for lesions accompanied by calcified nodules. ${ }^{8)}$ CCTA findings of positive remodeling or low-density plaque content do not necessarily characterize all of the plaques leading to ACS. In fact, no reports have classified the culprit plaques according to these CCTA characteristics.

In the present study we examined the CCTA features of 
plaques that progressed to acute myocardial infarction (AMI) in 31 consecutive patients in whom AMI developed more than 30 days after CCTA examination and in whom the culprit lesions were confirmed by coronary angiography.

\section{Methods}

\section{Study population}

From July 13, 2005 to March 31, 2012, our institution performed 64- or 320-slice CCTAs in 8,777 consecutive patients in whom coronary artery disease (CAD) was suspected on the basis of the following: chest symptoms, ECG abnormality, evidence of CAD in preoperative evaluation and one or more coronary risk factors. Of these patients, 373 were excluded because of unacceptable image quality. Based on the CCTA database of the remaining 8404 patients $(\mathrm{M} / \mathrm{F}=4582 / 3822$; 66 \pm 12 yrs; known CAD: 2319 patients), 31 consecutive patients $(\mathrm{M} / \mathrm{F}=29 / 2 ; 67 \pm 10 \mathrm{yrs}$; period to AMI onset: $559 \pm 490$ days (range: 52-1707), known CAD: 16 cases) in whom AMI developed more than 30 days after the CCTA examination and in whom culprit lesions were confirmed in our institution were enrolled. Patients whose culprit lesions were localized at previous stent site were excluded. We examined the culprit plaque characteristics on CCTA for AMI.

This retrospective study was approved by the ethics committee of our institution. Written informed consent from individual patients was waived.

\section{Definition of AMI}

AMI was defined as ST-segment elevation myocardial infarction (STEMI) and non-ST-segment elevation myocardial infarction (NSTEMI). Patients with ischemic discomfort, STsegment elevation and elevated troponin or creatine kinase were defined as STEMI. Patients who experienced ischemic discomfort and presented with elevated troponin or cratine kinase in the absence of ST-segment elevation on the ECG were defined as NSTEMI.

The culprit lesion in AMI was determined based on invasive coronary angiography, echocardiography, and ECG.

\section{64-slice MDCT protocol and 320-slice MDCT protocol}

CCTA images were acquired according to the protocol of our institution reported previously. ${ }^{9)}$

Briefly, scanning was performed using a 64-slice and 320slice MDCT scanner (Aquilion 64, Toshiba Medical Systems Corporation, Aquilion ONE V4.51, Toshiba Medical Systems Corporation), an ECG monitor (BSM-2401, Nihon Kohden), and an injector (Stellant Dual Flow, Nihon Medrad K.K.). A 3D workstation (Zio M900, Ziosoft) was used to generate 3-D (volume rendering) and curved planar reconstruction (CPR) images. The contrast medium injection time was fixed and the injection rate and injection volume were determined according to the patient's weight. Scanning was performed at a tube voltage of $120 \mathrm{kV}$ and a tube current of 400 to $600 \mathrm{~mA}$. For patients with a $\mathrm{HR} \geq 60 \mathrm{bpm}, 25 \mathrm{mg}$ of atenolol was administered orally on the night before the examination unless contraindications were present. Alternatively, 2 to $10 \mathrm{mg}$ of propranolol was injected intravenously immediately before scanning to obtain low HR. Steps were always taken to reduce the radiation dose to the greatest extent possible using prospective ECG-gated scanning. ${ }^{9)}$

\section{Image reconstruction}

Half image reconstruction or segmental image reconstruction was performed in the slow filling phase or end-systolic phase using the " $\mathrm{R}+$ absolute time" method to generate images. Images with the lowest level of motion artifacts were selected on the 4-chamber cardiac cine CT.

\section{CCTA interpretation}

Both cross-sectional and longitudinal curved multiplanar reformation images were analyzed for plaque detection. Coronary arteries were divided into 17 segments based on the recommendations of the modified American Heart Association. ${ }^{10)}$ Coronary artery segments with a diameter of $>2 \mathrm{~mm}$ were evaluated for the presence of plaques. The following characteristics were determined for all plaques: the presence of significant stenosis, the presence of vessel remodeling (positive, negative, none), plaque consistency (low-density plaque or not), and the disposition of coronary calcification (spotty, moderate, severe). Three observers interpreted the data.

\section{Definitions of the CT plaque characteristics}

Degree of stenosis: Visual assessment of the stenotic lesion was undertaken based on the AHA classification. The percentage ratio of the stenotic lumen to the original vessel diameter of the lesion analogized by a presumed-to-be-healthy site distal and proximal to the stenosis was determined and the degree of stenosis was expressed by subtracting this from 100 . Out of still images taken from multiple projections, measurements were performed from the angle showing the narrowest degree of stenosis in order to classify the lesion into four stages: $25 \%$ for stenosis of $0 \%-25 \%, 50 \%$ for $26 \%-50 \%, 75 \%$ for $51 \%-75 \%$ and $>75 \%$ for $76 \%-100 \%$. Lesions with stenosis of $75 \%$ or more were defined as significant stenotic lesions.

Vessel remodeling: Coronary arterial remodeling was defined as a change in the vessel diameter at the plaque site in comparison to the reference segment set proximal to the lesion in a normal-appearing vessel segment (reference diameter). The remodeling index (lesion diameter/reference diameter) was defined by manual inspection in both cross-section and longitudinal reconstruction. The remodeling index was reported as positive remodeling when the diameter at the plaque site was at least $10 \%$ larger than the reference segment. 
J Jpn Coron Assoc 2015; 21: 287-295

Table 1 Comparison of clinical characteristics among the various plaque types based on CCTA

\begin{tabular}{|c|c|c|c|c|c|}
\hline & Type I & Type II & Type III & Type IV & $\mathrm{P}$ value \\
\hline Number of patients & 17 & 7 & 4 & 3 & \\
\hline Positive remodeling & + & + & - & - & \\
\hline Low density plaque & + & - & - & - & \\
\hline severe calcification & - & - & - & + & \\
\hline Age & $64 \pm 8^{*}$ & $65 \pm 9^{\#}$ & $79 \pm 8$ & $74 \pm 6$ & $\mathrm{P}=0.0134$ \\
\hline Gender $(\mathrm{M} / \mathrm{F})$ & $16 / 1$ & $7 / 0$ & $3 / 1$ & $3 / 0$ & $\mathrm{P}=0.3949$ \\
\hline Family history (\%) & $1(5.9 \%)$ & $0(0 \%)$ & $0(0 \%)$ & $1(33.3 \%)$ & $\mathrm{P}=0.2252$ \\
\hline Hypertension (\%) & $9(52.9 \%)$ & $4(57.1 \%)$ & $1(25 \%)$ & $2(66.7 \%)$ & $\mathrm{P}=0.6813$ \\
\hline Dislipidemia (\%) & $14(82.4 \%)$ & $5(71.4 \%)$ & $4(100 \%)$ & $0(0 \%)$ & $\mathrm{P}=0.0139$ \\
\hline Diabetes $(\%)$ & $3(17.6 \%)$ & $2(28.6 \%)$ & $2(50 \%)$ & $0(0 \%)$ & $\mathrm{P}=0.3954$ \\
\hline Smoking (\%) & $5(31.3 \%)$ & $3(42.9 \%)$ & $1(25 \%)$ & $0(0 \%)$ & $\mathrm{P}=0.5942$ \\
\hline Period to onset (days) & $504 \pm 457$ & $629 \pm 511$ & $605 \pm 746$ & $651 \pm 507$ & $\mathrm{P}=0.9271$ \\
\hline Stenosis degree $(25 \% / 50 \% />75 \%)$ & $8 / 4 / 5$ & $4 / 2 / 1$ & $2 / 2 / 0$ & $1 / 1 / 0$ & $\mathrm{P}=0.8473$ \\
\hline Asprin $(\%)$ & $11(64.7 \%)$ & $6(85.7 \%)$ & $3(75.0 \%)$ & $2(66.7 \%)$ & $\mathrm{P}=0.7720$ \\
\hline Statin $(\%)$ & $3(17.6 \%)$ & $3(42.9 \%)$ & $0(0 \%)$ & $0(0 \%)$ & $\mathrm{P}=0.7720$ \\
\hline
\end{tabular}

* vs Type III $\mathrm{p}<0.005$

\# vs Type III $\mathrm{p}<0.05$

CCTA: coronary computed tomography angiography

Plaque consistency: If the axial image of the culprit plaque included areas of non-calcified plaque or spotty calcification and the consistency of the plaque could be measured, the image was used to measure the $\mathrm{CT}$ value. The $\mathrm{CT}$ value of the culprit plaque was defined as the minimum CT value of five pieces of minimum ROI $(0.36 \mathrm{~mm} \times 0.36 \mathrm{~mm})$. Low-density plaque was defined as plaque with $\mathrm{CT}$ values $\leq 50 \mathrm{HU}$.

Calcification: Plaque calcification was classified as spotty, moderate and severe. Spotty calcification was defined as a size of $<3 \mathrm{~mm}$ on curved multiplanar reformation images and appearance on only 1 side on cross-sectional images. ${ }^{2,3)}$ Moderate calcification was defined as calcification larger than spotty with a plaque circumference of $180^{\circ}$ or less. Severe calcification was defined as calcification larger than spotty with a plaque circumference of more than $180^{\circ}$.

\section{Classification of vulnerable plaque based on CCTA}

Vulnerable plaques were classified into four types based on the CCTA findings of positive remodeling, plaque density, and the presence of calcification (see Table 1 and Fig. 3-6). The types were classified as follows: Type I, positive remodeling $(+)$ and low-density $(+)$; Type II, positive remodeling $(+)$ and lowdensity (-); Type III, positive remodeling $(-)$ and low-density $(-)$; Type IV, positive remodeling $(-)$ and severe calcification $(+)$.

\section{Definition of risk factors}

Hypertension (HT) was defined as blood pressure $\geq 140 / 90$ $\mathrm{mmHg}$ or the use of antihypertensive treatment. Diabetes mellitus (DM) was defined as fasting blood sugar $\geq 126 \mathrm{mg} / \mathrm{dl}$, postprandial blood sugar $\geq 200 \mathrm{mg} / \mathrm{dl}$, hemoglobin A1c $\geq 6.5 \%$ or the use of blood sugar-lowering treatment with or without insulin. Dyslipidemia was defined as total cholesterol $\geq 220$ $\mathrm{mg} / \mathrm{dl}$, low-density lipoprotein cholesterol $\geq 140 \mathrm{mg} / \mathrm{dl}$, fasting triglycerides $\geq 150 \mathrm{mg} / \mathrm{dl}$, high density cholesterol $<40 \mathrm{mg} / \mathrm{dl}$ or the use of lipid-lowering treatment. Patients who had smoked in the 1 year leading up the CCTA examination were defined as smokers.

\section{Statistical analyses}

Statistical analyses were performed using Statview J-5.0 for Windows (HULINKS, Inc., Tokyo Japan). Numerical data were expressed as the mean \pm standard deviation. One factor ANOVA (for age and period to onset of ACS) or chi-square tests (for gender, family history, HT, dyslipidemia, DM, smoking, stenosis of degree value and medication value) were employed for comparisons between groups to determine the statistical significance of differences. Groups were compared independently using Bonferroni/Dunn analysis. Values at $\mathrm{p}<0.05$ were considered statistically significant in all instances.

\section{Results}

\section{Plaque characteristics of culprit lesion in 31 cases}

Culprit lesions were the LMT in two patients; RCA segments $1,2,3$, and $4 \mathrm{AV}$ in four, four, three, and one cases, respectively; LAD segments 6,7 , and 8 in eight, two, and two patients, respectively; and LCX segments 11, 12, and 13 in three, one, and two patients, respectively.

As for the degree of stenosis, 25\% stenosis was found in 15 cases, $50 \%$ was found in 9 , and $\geq 75 \%$ was found in 7 . No significant stenosis $(\leq 50 \%)$ was observed in 24 cases $(77.4 \%)$ (Fig. 1A).

Positive remodeling (remodeling index $>1.1$ ) was found in 24 

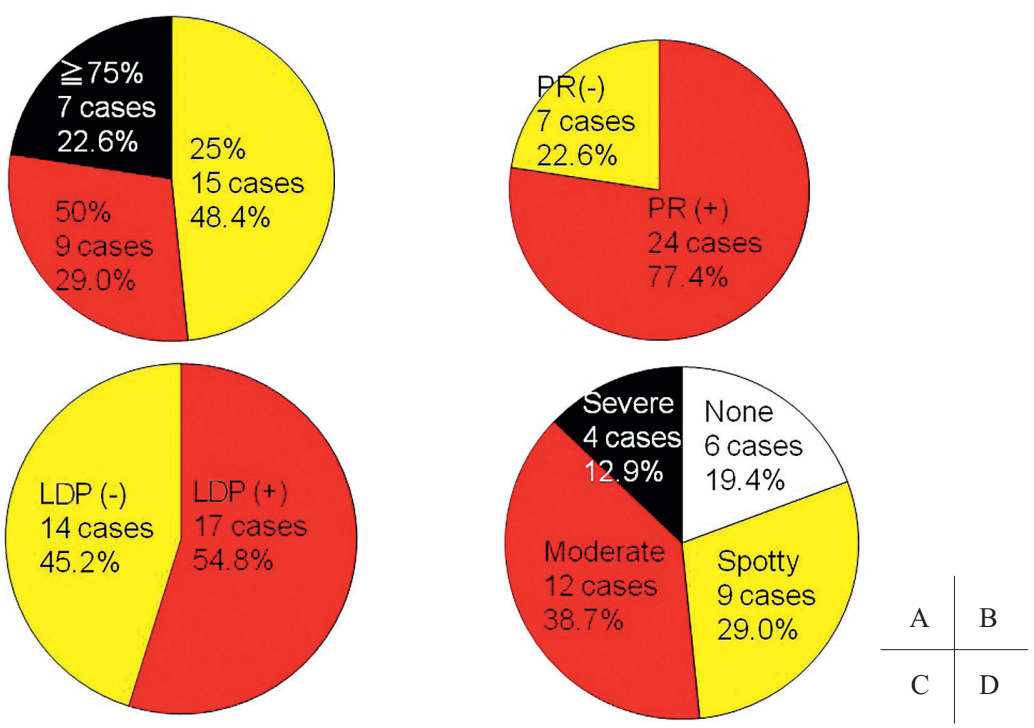

Fig. 1 Plaque characteristics of culprit lesion.

A: 25,50 and $>75 \%$ stenosis was found in 15,9 and 7 cases, respectively.

B: Positive remodeling was found in 24 cases $(77.4 \%)$.

C: Low-density plaques were found in 17 cases $(54.8 \%)$.

D: Different degrees of calcification were demonstrated in the culprit lesions: none, spotty, moderate, and severe in 6, 9, 12, and 4 cases, respectively.

PR: positive remodeling, LDP: low-density plaque

cases $(77.4 \%$ ) (average remodeing index 1.5 \pm 0.2 ) (Fig. 1B) and low-density plaques (CT values $\leq 50)$ were found in 17 cases $(54.8 \%)$ (Fig. 1C). Patients with positive remodeling were significantly younger than patients without positive remodeling $(\mathrm{p}<0.001$, data not shown).

Calcification was spotty in 9 cases, moderate in 12 , severe in 4, and absent in 6 (Fig. 1D).

Although the period from CCTA examination to AMI onset (days) tended to be shorter in $\geq 75 \%$ stenosis than in $25 \%$ stenosis, no significant differences among the values were observed ( $25 \%$ vs $50 \%$ vs $\geq 75 \%$ : $698 \pm 488$ vs $507 \pm 513$ vs 329 \pm 427 ) (Fig. 2).

2. Comparison of clinical characteristics between various vulnerable plaque types on CCTA

There were no significant differences in gender, coronary risk factors (family history, HT, DM, smoking), period to AMI onset, degree of stenosis or medication (aspirin and statin) among four groups. Age (type I vs II vs III vs IV $=64 \pm 8$ vs $65 \pm 9$ vs $79 \pm 8$ vs $74 \pm 6 ; \mathrm{p}=0.0134)$ and dyslipidemia $(82.4 \%$ vs $71.4 \%$ vs $100 \%$ vs $0 \%: \mathrm{p}=0.0139)$ were the only parameters showing significant differences among four groups (Table 1). Type I was significantly younger than the remaining types (Fig. 7).

\section{Discussion}

In the present study, we evaluated the CCTA findings of plaques in 31 consecutive patients in whom AMI developed after

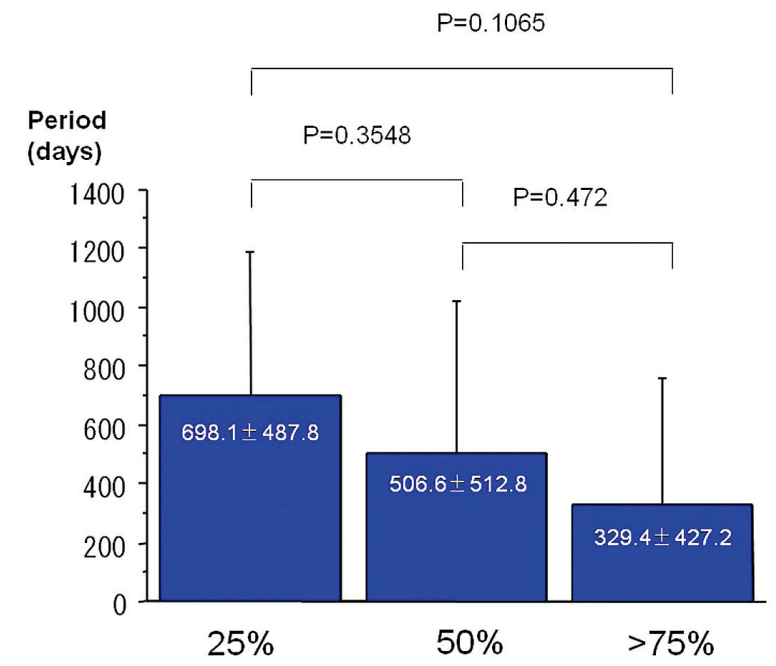

Fig. 2 Comparison of the period from the CCTA examination to the onset of AMI by degree of stenosis.

The period to AMI onset tended to be shorter in lesions with $>75 \%$ stenosis, but the difference was not significant.

AMI: acute myocardial infarction

an average of 559 days from the CCTA examination and in whom the culprit lesions were confirmed by coronary angiography. As a result, vulnerable plaques could be classified into four types according to the plaque characteristic on CCTA. To our knowledge, there have been no earlier reports on the CCTA classification or prevalence for each type of vulnerable 

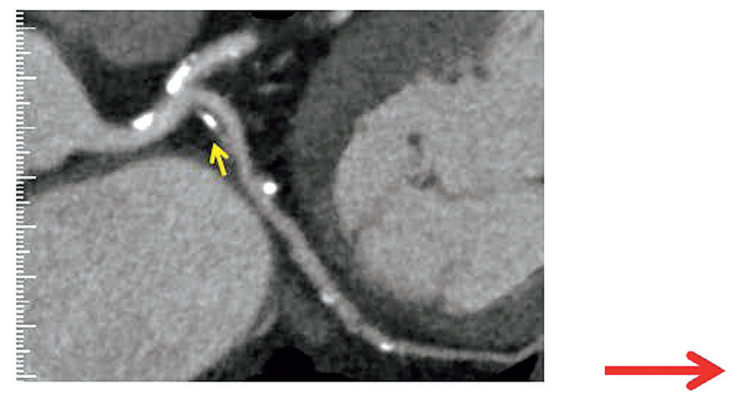

After 199 days
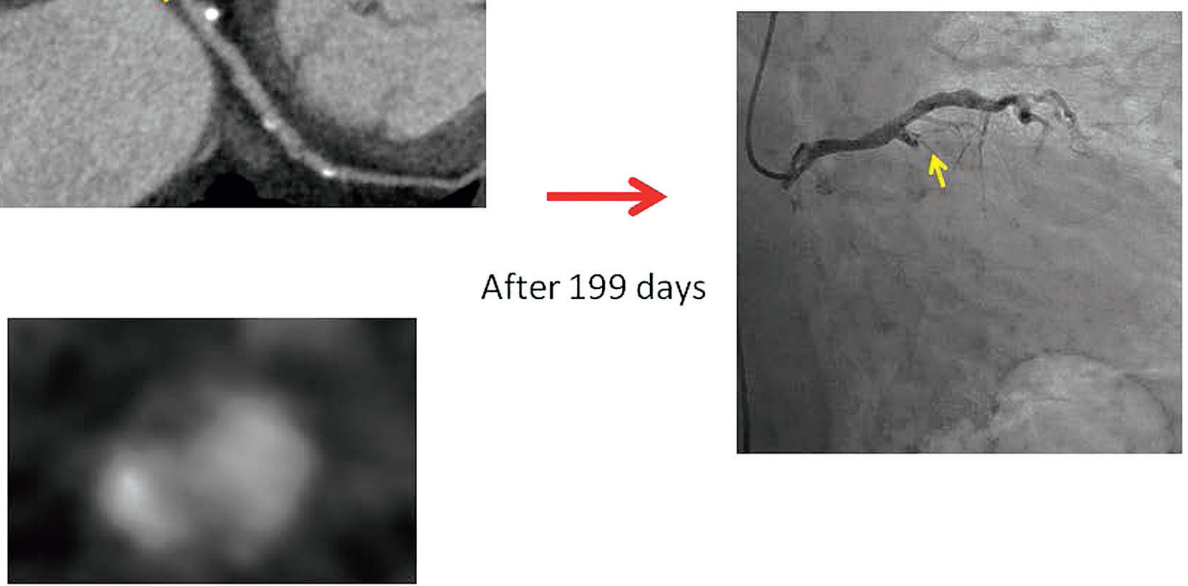

Fig. 3 Representative case of type I.

The patient was a 63 -year-old male with two coronary risk factors: HT and dyslipidemia. CCTA at our hospital revealed a $25 \%$ stenotic lesion with positive remodeling (+) (remodeling index 1.6), lowdensity plaque $(+)$, and moderate calcification at LCX\#11. The patient came to our hospital with a complaint of chest oppression 199 days after the CCTA examination. AMI was diagnosed on the basis of ST elevation in leads II, III, aVf and ST depression in V2-V4 on ECG. Emergency CAG revealed a total occlusive lesion in the locus accorded with type I plaque of LAD\#11 on CCTA.

HT: hypertension, CCTA: coronary computed tomography angiography, AMI: acute myocardial infarction, CAG: coronary angiography

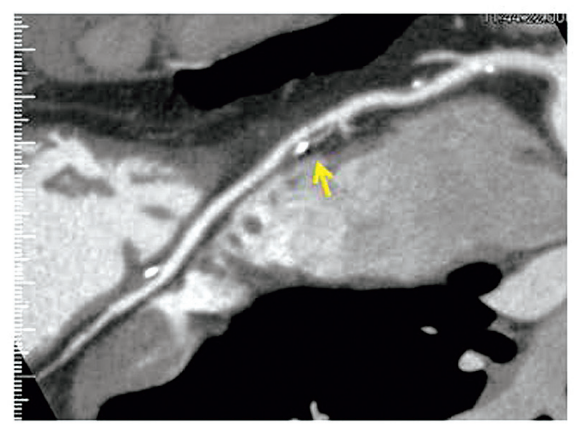

After 1535 days
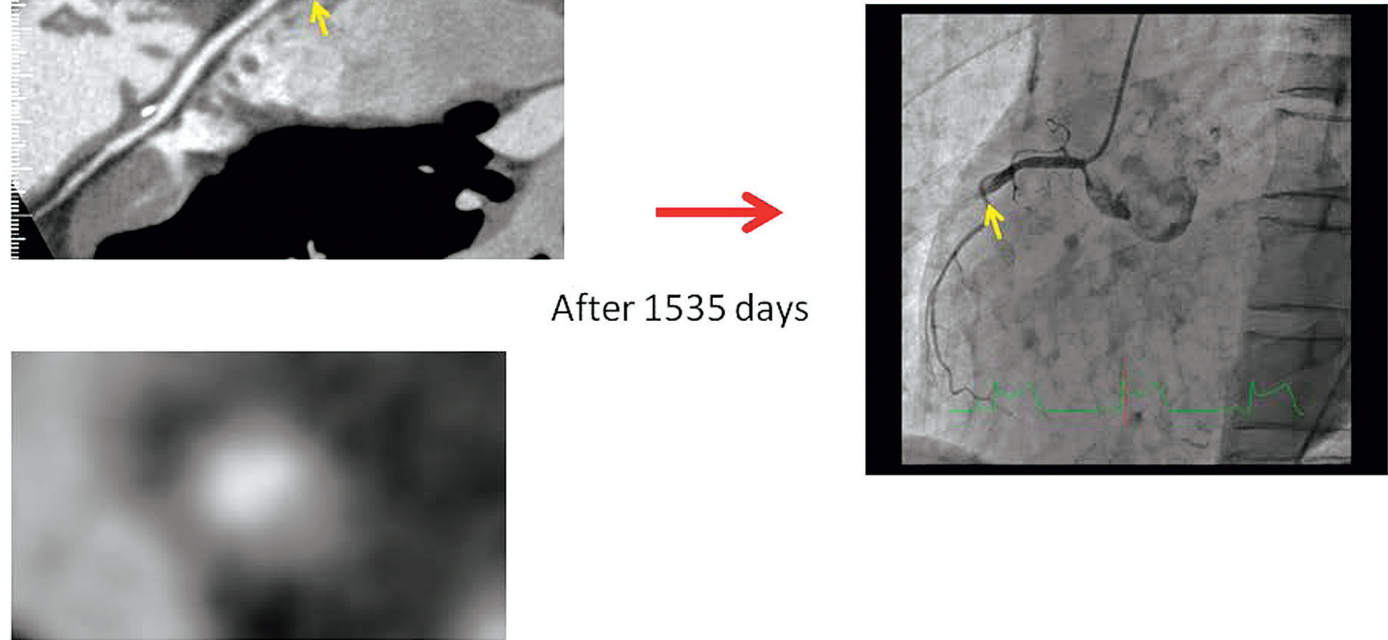

Fig. 4 Representative case of type II.

The patient was a 60 -year-old male with one coronary risk factor: dyslipidemia. CCTA at our hospital revealed a $50 \%$ stenotic lesion with positive remodeling (+) (remodeling index 1.7), low-density plaque (-), and moderate calcification at RCA\#2. The patient came to our hospital with a chief complaint of chest pain 1,535 days after the CCTA examination. AMI was diagnosed on the basis of ST elevation of leads II, III, aVf on ECG. Emergency CAG revealed a total occlusive lesion in the locus accorded with type II plaque of RCA\#2 on CCTA.

CCTA: coronary computed tomography angiography, AMI: acute myocardial infarction, CAG: coronary angiography 

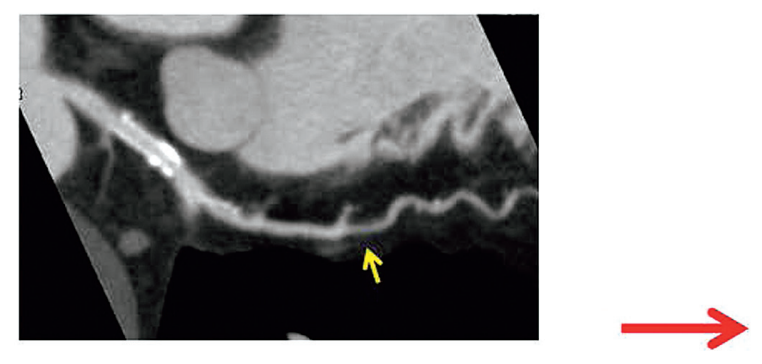

After 373 days
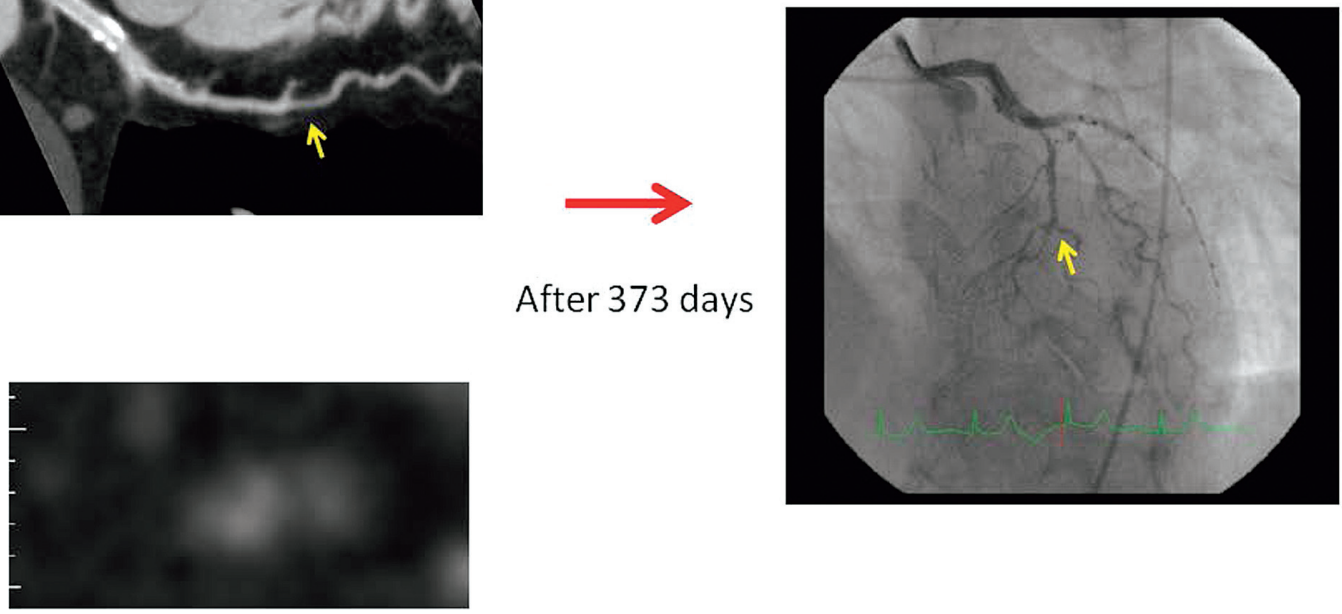

Fig. 5 Representative case of type III.

The patient was a 70-year-old male with four coronary risk factors: HT, dyslipidemia, DM, and smoking. The patient underwent CCTA at our hospital to follow a lesion treated by percutaneous coronary intervention. CCTA findings showed a $50 \%$ stenotic lesion with positive remodeling (-), low-density plaque (-), and spotty calcification at LAD\#8. The patient came to our hospital with a chief complaint of chest pain 373 days after the CCTA examination. AMI was diagnosed based on ST elevation of V2-V5 on ECG. Emergency CAG revealed a delayed 99\% stenotic lesion in the locus accorded with type III plaque of LAD\#8 in CCTA.

HT: hypertension, DM: diabetes mellitus, CCTA: Coronary computed tomography angiography, AMI: acute myocardial infarction, CAG: coronary angiography
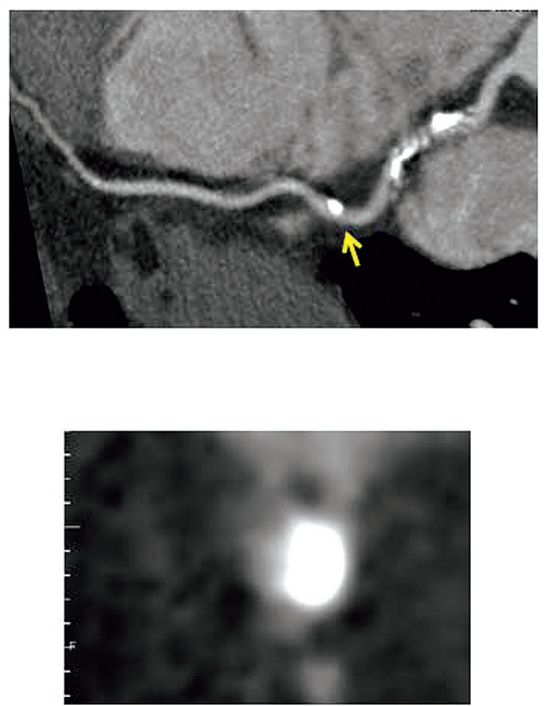

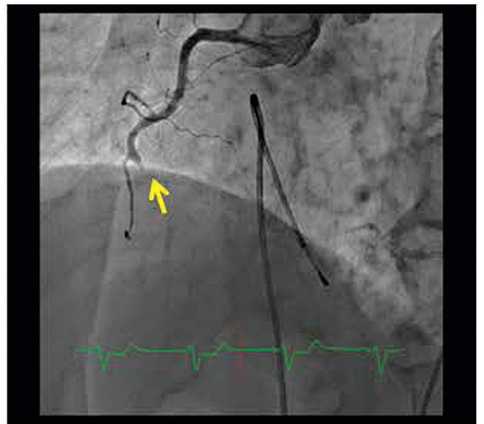

Fig. 6 Representative case of type IV.

The patient was a 67-year-old male with one coronary risk factor: a family history. CCTA at our hospital revealed a $25 \%$ stenotic lesion with severe calcification at RCA\#3. The patient came to our hospital with a chief complaint of chest pain 932 days after the CCTA examination. AMI was diagnosed based on ST elevation of leads II, III, and aVf on ECG. Emergency CAG revealed a total occlusive lesion in the locus accorded with type IV plaque of RCA\#3 in CCTA.

CCTA: coronary computed tomography angiography, AMI: acute myocardial infarction 


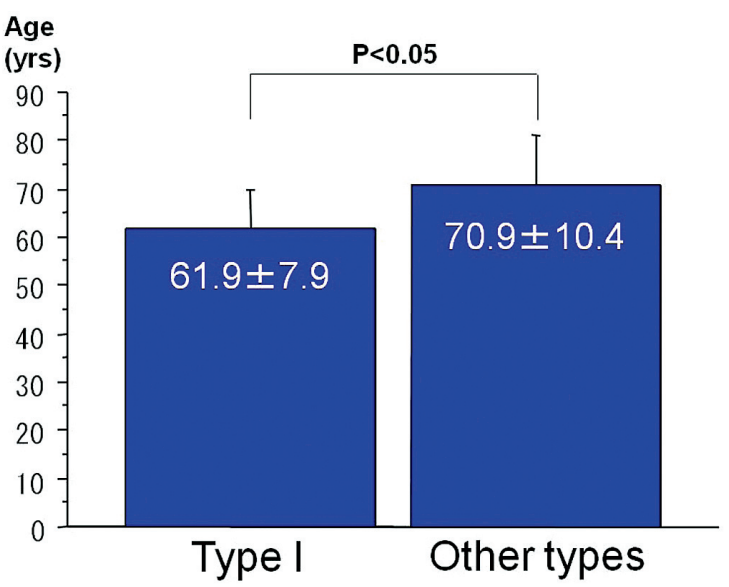

Fig. 7 Age comparison between type I and other types. Type I patients were significantly younger than the patients of the other types.

plaques.

The onset of AMI is difficult to predict. Many patients who develop the disease do so without precursor symptoms. A metaanalysis of coronary angiography findings revealed that $68 \%$ of coronary lesions that went on to cause ACS were free of significant stenosis. ${ }^{11)}$ The culprit lesions in ACS are also reported to be distributed predominantly in the proximal portions of the three major coronary arteries. ${ }^{12-14)}$ The distribution of culprit lesions in the present study was similar to the distributions reported from the three earlier studies. ${ }^{12-14)}$ Further, the prevalence of lesions without significant stenosis $(<75 \%)$ in our CCTA findings (77.4\%) was slightly higher than that previously reported. ${ }^{11)}$ This may be partly explained by the more aggressive revascularization now performed for lesions with significant stenosis $(>75 \%)$. Although the period to ACS onset tended to be shorter in lesions with $>75 \%$ stenosis, there was no significant difference. With the progression of stenosis, a patient may be more likely suffer symptomatic ACS induced by coronary occlusion with thrombi. Pathologically, $78 \%$ of acute rupture plaques manifested $\geq 50 \%$ narrowing in the crosssectional area. ${ }^{15)}$ A recent prospective study using virtual histology intravascular ultrasound showed that a minimal lumen area of $4.0 \mathrm{~mm}^{2}$ or less in a culprit plaque was predictive of the development of ACS. ${ }^{16)}$ The degree of stenosis in culprit plaque may progress between the CCTA examination and ACS onset in some cases.

The plaques responsible causing AMI in the present study were classified into four types, (Types I to IV), according to the CCTA characteristics. Type I plaque characterized by positive remodeling and low-density plaque content was the most frequent $(54.8 \%)$. Plaque with positive remodeling and lowdensity plaque content on CCTA has been regarded as ruptureprone vulnerable plaque. Motoyama et al. reported that culprit lesions had significantly more positive remodeling and lowattenuation plaque than lesions of stable angina pectoris in ACS cases. ${ }^{2)}$ Importantly, patients with two-feature positive plaque, that is, plaque characterized by positive remodeling and lowattenuation plaque content, are reported to have a $22 \%$ probability of developing ACS within 2 years. ${ }^{3)}$ It has been reported that $55-60 \%$ of ACS cases resulting from luminal thrombi are induced by plaque rupture pathologically. ${ }^{2)}$ The frequency of Type I plaque, a plaque prone to rupture, was almost the same in the ACS cases in that report as in the cases we report here. ${ }^{2)}$ However, rupture-prone vulnerable plaques could also be classified as Type II plaques with positive remodeling and no low-density plaque content. A low CT value is thought to reflect a soft, lipid-rich plaque. Yet CT values are affected by the level of contrast media in the coronary lumen, the degree of stenosis in the coronary lesion, and the tube voltage. ${ }^{17}$ The presence of calcification in the peripheral area may also affect the CT value of a plaque in some cases, even if an area of non-calcified plaque with a measurable CT value is present in the axial image. The average CT values of soft, fibrous, and calcified plaques evaluated by IVUS were $11 \pm 12 \mathrm{HU}, 78 \pm 21$ HU, $516 \pm 198 \mathrm{HU}$, respectively, in 0.5-mm-slice MDCT. ${ }^{18)}$ Thus, a CT value of less than $30 \mathrm{HU}$ was concluded to be proper for soft plaque. Pohle et al. ${ }^{19)}$ found a significant difference in the CT values of hypo-echogenic plaques $(58 \pm 43 \mathrm{HU})$ and hyperechogenic (121 $\pm 39 \mathrm{HU})$ plaques, but overlapping CT values precluded a CT-based division of those plaques. Another study comparing CCTA and autopsy findings demonstrated that the average $\mathrm{CT}$ attenuation of napkin-ring plaques (approximately 50 to $60 \mathrm{HU}$ ) was higher than the previously suggested cutoff value (30 HU) for high-risk lesions. ${ }^{20)}$ The cut-off CT value used in the present study was less than $50 \mathrm{HU}$. Further research to determine the cutoff $\mathrm{CT}$ value for lipid-rich plaques is awaited. In addition, other plaque types at the baseline might change to Type I plaques during the observation period. A previous report of IVUS findings demonstrated that thick-cap fibroatheromas changed into thin-cap fibroatheromas. ${ }^{21)}$

Pathologically, coronary occlusion with thrombi is caused by erosion in $30-35 \%$ of cases and by calcified nodules in $3-7 \% .^{8}$ Plaque erosion is defined as the plaque causing an acute thrombus in direct contact with the intima in an area denuded of endothelium. The plaque content is rich in smooth muscle cells and proteoglycan matrix. ${ }^{22)}$ Plaque erosion and plaque rupture both occur mainly in the proximal portion of the coronary artery, but the patients with erosion tend to be significantly younger than the patients with rupture, on average. ${ }^{22)}$ Some Type II plaque may lead to the development of AMI because of plaque erosion. The patients with Type III plaque were significantly older than the patients in the other three groups, and all of them 
were dyslipidemic. Moreover, Type III plaque occurred predominantly in the distal portion of the coronary artery. Therefore, there is no conclusive evidence that Type III plaque is similar to plaque erosion. Because plaque erosion also occurred predominantly in the proximal portion of the coronary artery and is associated with smoking especially in women. Individuals with erosion are younger than those with rupture. CCTA findings from the present study showed no discernable similarity between plaque erosion and any of the other plaque types. In a recent study by Ozaki et al., CCTA failed to characterize culprit lesions assessed as plaque erosion by optical coherence tomography. ${ }^{23)}$

Type IV plaque, that is, plaque with severe calcification, was observed in 3 cases $(11.1 \%)$ in the present study. Some Type IV plaques were thought to induce AMI via the growth of calcified nodules (ACS is caused by calcified nodules in 3-7\% of cases overall), as most of the calcified nodules observed were present in calcified plaque. Yet thin-cap fibroatheroma has also been seen in heavily calcified areas, which suggests that the calcification pattern is unhelpful for diagnosing these lesions. ${ }^{24)}$ As such, rupture-pone vulnerable plaque might not be identifiable solely on the basis of severe calcification in CCTA. By general consensus, most vulnerable plaques have no calcification, or only microcalcification. ${ }^{7)}$ Yet in our clinical experience, AMI develops at the sites of culprit lesions with severe calcification. The present study demonstrated that even plaque with severe calcification could cause AMI.

The plaque components reported to be most strongly associated with positive remodeling are macrophage infiltration and high percentages of calcium and lipid core. ${ }^{25)}$ Positive remodeling has been defined as compensatory vessel enlargement to counter absence or loss of lumen in case where crosssectional luminal narrowing of $40 \%$ or more is observed. ${ }^{26,27)}$ We were thus unsurprised to find, in the present study, that the patients with both Type I and II plaques with positive remodeling were significantly younger than the patients in the other groups.

\section{Limitation}

There are some limitations in the present study. First, only 31 consecutive patients in whom culprit lesions were confirmed in our institution were enrolled in the present study. We were unable to observe outcome for all of 8,404 consecutive patients who underwent CCTA. Therefore, only $0.32 \%$ of the 8,404 patients in total were subjects for this study. Second, the degree of stenosis in the present study was evaluated not by vessel area but by vessel diameter. Third, CT values are influenced by factors such as the concentration of contrast medium in the coronary artery, the degree of coronary lesion stenosis, and tube voltage. We are unable to validate the cutoff $\mathrm{CT}$ value used for our analysis (less than $50 \mathrm{HU}$ ), as medical consensus on the cutoff $\mathrm{CT}$ value has yet to be reached. ${ }^{17)}$

\section{Conclusions}

Vulnerable plaque in CCTA could be classified into four types based on the plaque characteristics. Type I plaque (positive remodeling $(+)$ and low-density plaque $(+))$, the type assessed as thin cap fibroatheroma on CCTA, was the most frequent (54.8\%) and was associated with a younger patient age. Further study on the prediction of AMI using CCTA is awaited.

\section{References}

1) Narula J, Finn AV, Demaria AN: Picking plaques that pop ... J Am Coll Cardiol 2005; 45: 1970-1973

2) Motoyama S, Kondo T, Sarai M, et al: Multislice computed tomographic characteristics of coronary lesions in acute coronary syndromes. J Am Coll Cardiol 2007; 50: 319-326

3) Motoyama S, Sarai M, Harigaya $H$, et al: Computed tomographic angiography characteristics of atherosclerotic plaques subsequently resulting in acute coronary syndrome. J Am Coll Cardiol 2009; 54: 49-57

4) Henneman MM, Schuijf JD, Pundziute G, et al: Noninvasive evaluation with multislice computed tomography in suspected acute coronary syndrome: plaque morphology on multislice computed tomography versus coronary calcium score. J Am Coll Cardiol 2008; 52: 216-222

5) Kashiwagi M, Tanaka A, Kitabata $\mathrm{H}$, et al: Feasibility of noninvasive assessment of thin-cap fibroatheroma by multidetector computed tomography. JACC Cardiovasc Imaging 2009; 2: 1412-1419

6) Narula J, Achenbach S: Napkin-ring necrotic cores: defining circumferential extent of necrotic cores in unstable plaques. JACC Cardiovasc Imaging 2009; 2: 1436-1438

7) Naghavi M, Libby P, Falk E, et al: From vulnerable plaque to vulnerable patient: a call for new definitions and risk assessment strategies: Part I. Circulation 2003; 108: 1664-1672

8) Virmani R, Burke AP, Farb A, et al: Pathology of the vulnerable plaque. J Am Coll Cardiol 2006; 47: C13-18

9) Fujimoto $S$, Matsutani $H$, Kondo $T$, et al: Image quality and radiation dose stratified by patient heart rate for coronary 64 - and 320-MDCT angiography. Am J Roentgenol 2013; 200: 765-770

10) Raff GL, Abidov A, Achenbach S, et al: SCCT guidelines for the interpretation and reporting of coronary computed tomographic angiography. J Cardiovasc Comput Tomogr 2009; 3: 122-136

11) Falk E, Shah PK, Fuster V: Coronary plaque disruption. Circulation 1995; 92: 657-671

12) Kolodgie FD, Burke AP, Farb A, et al: The thin-cap fibroatheroma: a type of vulnerable plaque: the major precursor lesion to acute coronary syndromes. Curr Opin Cardiol 2001; 16: 285-292

13) Wang JC, Normand SL, Mauri L, et al: Coronary artery spatial 
distribution of acute myocardial infarction occlusions. Circulation 2004; 110: 278-284

14) Hong MK, Mintz GS, Lee CW, et al: The site of plaque rupture in native coronary arteries: a three-vessel intravascular ultrasound analysis. J Am Coll Cardiol 2005; 46: 261-265

15) Virmani R, Burke AP, Kolodgie FD, et al: Vulnerable plaque: the pathology of unstable coronary lesions. J Interv Cardiol 2002; 15: 439-446

16) Stone GW, Maehara A, Lansky AJ, et al: A prospective naturalhistory study of coronary atherosclerosis. N Engl J Med 2011; 364: $226-235$

17) Cademartiri F, Mollet NR, Runza G, et al: Influence of intracoronary attenuation on coronary plaque measurements using multislice computed tomography: observations in an ex vivo model of coronary computed tomography angiography. Eur Radiol 2005; 15: 1426-1431

18) Motoyama $\mathrm{S}$, Kondo $\mathrm{T}$, Anno $\mathrm{H}$, et al: Atherosclerotic plaque characterization by $0.5-\mathrm{mm}$-slice multislice computed tomographic imaging. Circ J 2007; 1: 363-366

19) Pohle K, Achenbach S, Macneill B, et al: Characterization of non-calcified coronary atherosclerotic plaque by multi-detector row CT: comparison to IVUS. Atherosclerosis 2007; 190: 174180

20) Maurovich-Horvat P, Hoffmann U, Vorpahl M, et al: The napkin-ring sign: CT signature of high-risk coronary plaques?
JACC Cardiovasc Imaging 2010; 3: 440-444

21) Kubo $T$, Maehara A, Mintz GS, et al: The dynamic nature of coronary artery lesion morphology assessed by serial virtual histology intravascular ultrasound tissue characterization. J Am Coll Cardiol 2010; 55: 1590-1597

22) Farb A, Burke AP, Tang AL, et al: Coronary plaque erosion without rupture into a lipid core. A frequent cause of coronary thrombosis in sudden coronary death. Circulation 1996; 93: 1354-1363

23) Ozaki Y, Okumura $M$, Ismail $T F$, et al: Coronary $C T$ angiographic characteristics of culprit lesions in acute coronary syndromes not related to plaque rupture as defined by optical coherence tomography and angioscopy. Eur Heart J 2011; 32: 2814-2823

24) Burke AP, Weber DK, Kolodgie FD, et al: Pathophysiology of calcium deposition in coronary arteries. Herz 2001; 26: 239-244

25) Burke AP, Kolodgie FD, Farb A, et al: Morphological predictors of arterial remodeling in coronary atherosclerosis. Circulation 2002; 105: 297-303

26) Glagov S, Weisenberg E, Zarins CK, et al: Compensatory enlargement of human atherosclerotic coronary arteries. N Engl J Med 1987; 316: 1371-1375

27) Clarkson TB, Prichard RW, Morgan TM, et al: Remodeling of coronary arteries in human and nonhuman primates. JAMA 1994; 271: 289-294 